\title{
Empirical Study on Traffic Simulation Model in the Perspective of Raipur City
}

\author{
Sanjiv Kumar Shukla \\ Department of Computer Science and Engineering, \\ Rungta College of Engineering and Technology, \\ Bhilai, India
}

\begin{abstract}
Road network management is an important task for the economic and social growth. In the context of an Indian city, efficient road network management is required to overcome the problem of traffic congestions and road accidents. Computer traffic simulation is a better way to analysis the road network. In this study a maiden attempt for a systematic empirical traffic simulation study is performed in the perspective of Raipur city in India. The aim of this study is to set up, validate and experiment a simulation model of traffic by using Simulation of Urban Mobility (SUMO).
\end{abstract}

\section{General Terms}

Computer Simulation, Road Network Management, Modelling.

\section{Keywords}

Road congestion, Traffic Management, Urban Traffic Network, Traffic flow.

\section{INTRODUCTION}

Computer simulation of traffic is extensively utilized in traffic modelling, planning and development of traffic networks and systems research. Now in these days, it becomes an important tool to evaluate traffic management actions and to predict traffic. Vehicular traffic systems are of growing concern and interest globally and modelling complex traffic systems is a hard problem [1]. Computer traffic simulation provides a better understanding to manage the road traffic and it helps to increase the efficiency of road traffic and overcome the problem of traffic congestion. The particulate design road traffic management is the primary cause for extended periods of traffic congestion throughout [2]. As per the joint study of Transport Corporation of India and Indian Institute of Management Calcutta estimated the India loses nearly 600 billion of Indian Rupees a year due to traffic congestion, slow speed of freight vehicles and waiting time at toll plazas and checking points etc. [3]. Another concern about road traffic management is safety. Road safety is an issue of national concern, considering its magnitude and gravity and the consequent negative impacts on the economy, public health and the general welfare of the people. Today, Road Traffic Injuries are one of the leading causes of deaths, disabilities and hospitalizations, with severe socioeconomic costs, across the world. The reported total number of road accidents in India was 4, 86, 476 during year 2013 and 1, 37, 572 persons killed and 4, 94,893 people injured in those accidents [4]. It depicts that good traffic management is necessary for economic and social developments and computer simulation provide environments to test scientific models in order to prove or disprove their feasibility and correctness. Later on the computer simulated model can be applied for real time traffic analysis [1].

Computer scientist classified the road traffic model into three categories, namely macroscopic, microscopic simulation and

\author{
Anupam Agrawal \\ Department of Computer Science and Engineering, \\ Rungta College of Engineering and Technology, \\ Bhilai, India
}

mesoscopic model. A macroscopic model is utilized to simulate traffic flow by considering traffic characteristics such as speed, density, flow, and their relationships to each other. A microscopic model provides simulation of the traffic in a high level of detail. This model uses the movement of individual vehicle travelling along the road and the interaction between them. A mesoscopic model is a combination between microscopic and macroscopic simulation which has the ability to model a large study area with more detail information than the macroscopic model. While the microscopic model simulates real traffic behaviour with higher accuracy than mesoscopic model due to requirement of more detailed data in a microscopic model [5].

India is a fast growing country and one of the largest road networks of over $64,99,000 \mathrm{~km}$ in India [6]. Also, the medium cities are expanding as metro city. Government of India has launched a motivated 100 smart cities program and it is expected to expand of small cities and road networks. The efficient road network management and handle the traffic congestion will be major issues with the expansion of urban areas. Most of available literatures show that traffic simulation was performed in city developed countries. Only very few traffic simulation study was performed in Indian city compare as the number of cities in India. It gives opportunity to work in this field. The aim of this study is to set up, validate and experiment a simulation model of traffic in Raipur, Chhattisgarh by using Simulation of Urban Mobility (SUMO).

\section{MATERIAL AND METHODS}

\subsection{Study Area}

The traffic simulation was performed on the street network of Raipur. Raipur situates geographically in $21.21 \mathrm{oN}, 82.30 \mathrm{oE}$. Raipur is a city, the state of Chhattisgarh, India. It is the capital city of the state of Chhattisgarh. The total habitant of this city is nearly 112, 2555 as per Census of India 2011 [7]. Raipur connected through National highway such as NH6, NH43, NH 200, NH 217, NH 12A to various cities and also Asian highway AH 46 passes through this city. Raipur city map is shown in the Fig. 1.

\subsection{Simulation Tools}

In this study Simulation of Urban Mobility (SUMO) traffic simulation tool is used. The German Aerospace Center developed this traffic simulation tool and it is an open source, highly portable, microscopic road traffic simulation package. SUMO is licensed under the GPL. SUMO helps to prepare and perform the simulation of a traffic scenario along with traffic simulation [8]. SUMO utilizes a variant of Gipps car model namely Karuss car model for the simulation in default. These models are based on safety conditions derived from braking distances of the individual vehicle. In Krauss model, 


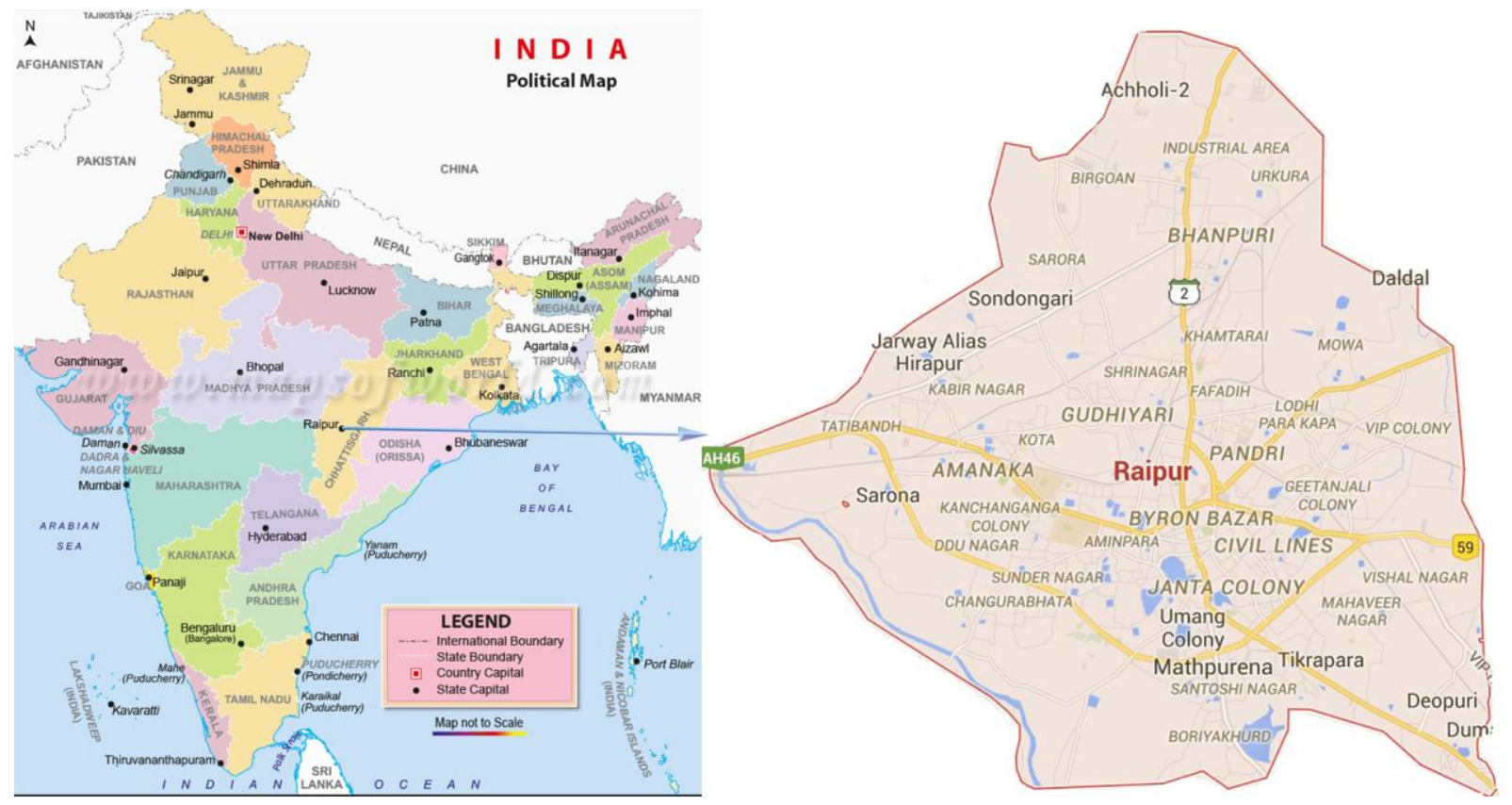

Fig 1: Map of Raipur, Chhattisgarh, India

$v_{\text {safe }}=v_{n+1}(t)+\frac{g_{n}(t)-v_{n-1}(t) \tau}{\frac{v_{n}(t)+v_{n+1}(t)}{2 b}+\tau}$

Where:

$\operatorname{gn}(\mathrm{t})=\mathrm{x}_{\mathrm{n}-1}(\mathrm{t})-\mathrm{x}_{\mathrm{n}}(\mathrm{t})-\mathrm{s}$

vdes $\leftarrow \min \{\mathrm{vn}(\mathrm{t})+\mathrm{a} . \Delta \mathrm{t}$, vsafe, vmax $\}$

$\operatorname{vn}(\mathrm{t}+\Delta \mathrm{t}) \leftarrow \max [0, \operatorname{rand}\{\mathrm{vdes}-\epsilon \mathrm{a}, \mathrm{vdes}\}]$

The position of the vehicle is denoted by index $\mathrm{n}$ and predecessor vehicle has higher index. So, in the traffic, leading vehicle has the highest index. The maximum acceleration (a), deceleration (b), and jammed spacing (s) are assumed constant. The distance between two vehicle is denoted by gn(t), vehicle desired speed (vdes) expresses how big the desire of drivers to accelerate their vehicle with a maximum speed, and the time step $(\Delta t)$ is assumed to be equal to the reaction time $(\tau)$. In the randomization step, each vehicle will be slowed down by random amount that is distributed between 0 and $\epsilon$, where $\epsilon$ is a value between 0 and 1 [5] [9].

For the proper run the simulation on SUMO, the four steps are required to perform. In the first step, the network building is required in the XML format. It includes the road network, intersection or junction and traffic lights. In the second step, demand file is required to create. It includes the physical property of vehicles such as type of vehicle, acceleration, deceleration, the length of the vehicle and maximum speed. In the third steps, the computation of dynamic use assignment is required. Dynamic user assignment is a traffic assignment which the assumption of the OD-matrix and the link flows are to be time dependent. It also simulates updated route choice. The last step is to run the simulation. The simulation can be run if the file contains the network, the information of routes that will be used in the simulation, the time period when the simulation will begin and end. With all information, the simulation can be performed in order to get a base model that will be applied to the simulation scenario [5] [8].
Additionally, NETCONVERT tool is used in this study for the generating road network from the XML file. NETCONVERT is a command line tool and it is developed in $\mathrm{C}++$ language. It can import digital road networks from different sources and generates road networks that can be used by other tools including SUMO [10]

\subsection{Data Collection and Preparation}

SUMO tool requires data set such as Road geometry of the city, Traffic flow and turning movement, Queue length of each section in a certain time period, Travel time data which is used for calibration. Open Street Map website [11] was utilized to obtain the road geometry data of Raipur city. The Open Street Map website provides facility to save street data on OSM file format. The .OSM file format is specific to Open Street Map. It is coded in XML and contains geographical data in a structured, ordered format [11]. Open Street Map contains only the street structure for the Raipur city. Therefore, the Google map [13] was used to validate and for proper naming of the street. SUMO uses the road network structure file in the XML file format; hence, NETCONVERT [10] tool was utilized to convert OSM files into road network structure file format. Fig. 2 shows the road network structure which was extracted from open street map and converted as desired format. Fig.3 depicts the closer view of this road network and the connection/junction of the roads is represented in the red colour box in this figure. Traffic flow data were collected by observation of the study location. In this regards, the major entry and exit points were identified and data were collected during the rush hours 9:30 AM to 11:00 and 17:00 19:00. The overall traffic flow was estimated based on that. Similarly, queue length and travel time data were collected. The route data is also required for the simulation on SUMO which contains a basic property of the vehicles such as length, acceleration and deceleration, and maximum speed of the vehicle. In this regards standard properties of vehicles were considered. 


\section{EXPERIMENT AND RESULT}

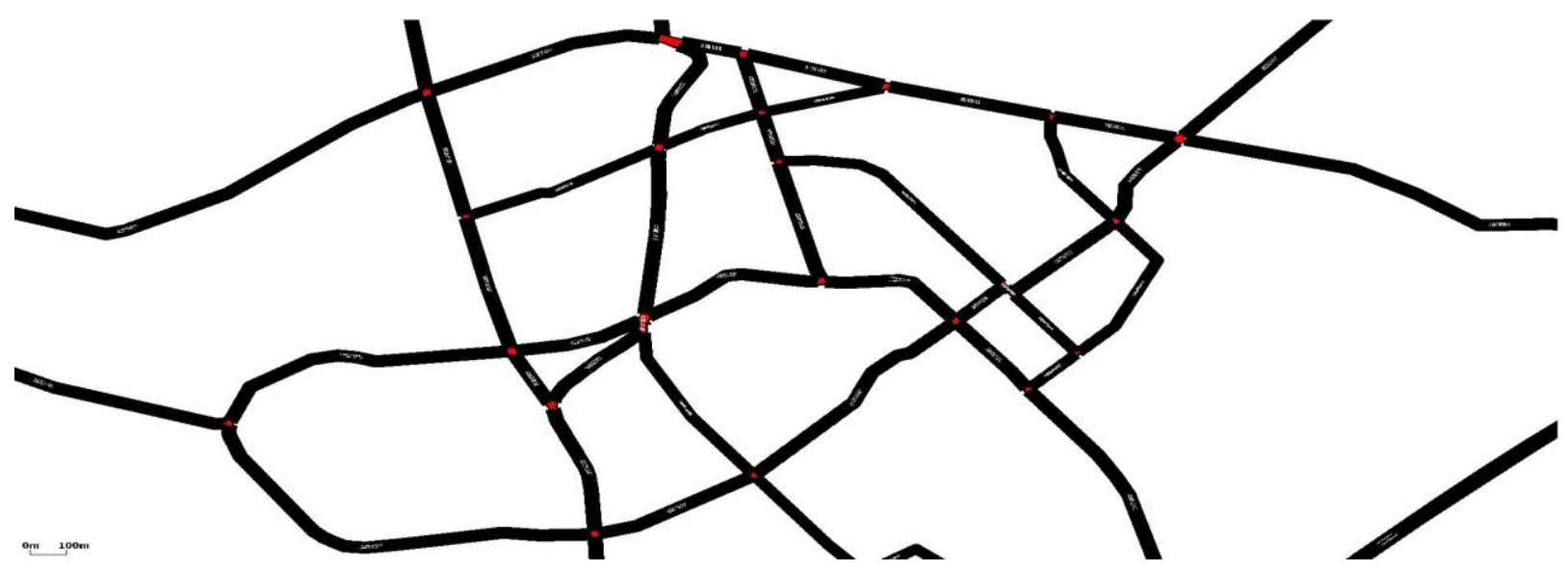

Fig 2: Street map of Raipur obtained from Open Street Map

The simulation was performed in SUMO version 0.22 .0 by considering the above mentioned data set. In the first step network was built. In the second step demand modeling was performed in this regards vehicle characteristics were supplied. In the last step configuration file was developed. It contains the network file and demand file. This file was saved with extension of sumo.cfg. This configuration file was supplied to SUMO to observe the simulation result. Further,

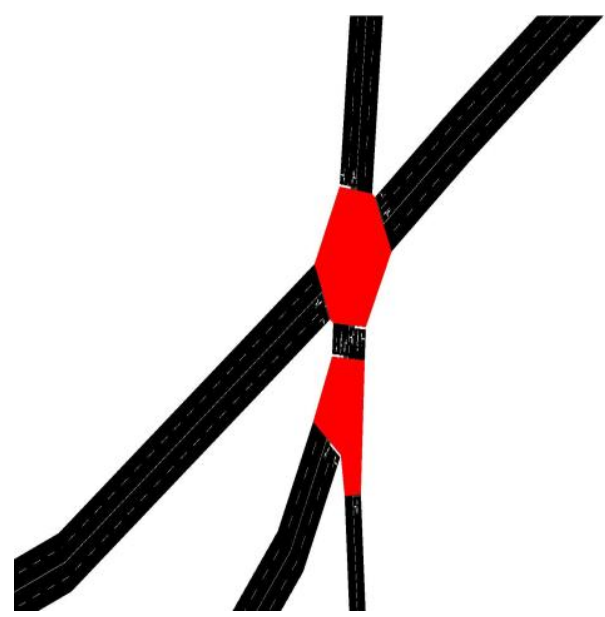

Fig 3: Closer view of Street map

calibration process was performed to identify the possible better parameters. It can be several parameters which will adjust to find the optimal value in trail process. It can be used for the allowable minimum distance between two vehicles, maximum acceleration, road speed limit (sigma), and the drivers' reaction time (tau). The initial model before the adjustment parameter is taken from the Maciejewski[14] research work for the calibration purpose and it is shown in the Table 1. Further, alternative scenario was developed and it was found traffic congestion, reduced when the some traffic diverts into AH46 high rather than GE Road. Similarly, if we provide a connected road between NH 30 and NH 53 the travel time also reduced.
Table 1. Initial model before adjustment

\begin{tabular}{|c|c|c|c|c|c|c|c|}
\hline $\begin{array}{l}\text { V } \\
\text { Type }\end{array}$ & $\begin{array}{l}\text { Le } \\
\mathbf{n} \\
(\mathrm{m})\end{array}$ & $\begin{array}{l}\text { Speed } \\
(\mathrm{m} / \mathrm{s})\end{array}$ & $\begin{array}{l}\text { Min } \\
\text { Gap } \\
\text { (m) }\end{array}$ & $\begin{array}{l}\text { Car } \\
\text { Folllowi } \\
\text { ng }\end{array}$ & $\begin{array}{l}\text { Max } \\
\text { Acce } \\
\text { l } \\
\left(\mathrm{m} / \mathrm{s}^{2}\right. \\
)\end{array}$ & $\begin{array}{l}\text { Sig } \\
\text { ma }\end{array}$ & $\begin{array}{l}\text { Ta } \\
\mathbf{u} \\
\text { (s) }\end{array}$ \\
\hline Car & 5 & 27.78 & 2 & Krauss & 5.50 & 0.50 & 1 \\
\hline Bus & 15 & 27.78 & 3 & & 5.50 & 0.50 & 1 \\
\hline Truck & 10 & 27.78 & 3 & & 5.50 & 0.50 & 1 \\
\hline
\end{tabular}

\section{CONCLUSION}

Efficient traffic management is needed in the present Indian scenario. Computer simulation on traffic management can provide a scenario of traffic and give idea to manage the traffic efficiently. In this study, the authors used SUMO traffic simulator to model the traffic of an urban city and analysis the performance. The study shows that the traffic simulator can be used to manage vehicle movement on the road network in order to avoid road congestion. This is a primary study to observe the feasibility of SUMO for the semi metro city and our expansion our study to incorporating the more demands and consider the all possible road networks. Further, mostly existing computer simulation model deals with Car and Bus vehicles. In the Indian context other vehicles are also used on the road such as Auto Rickshaws, Man pulled Rickshaws, Motorbike also some time roaming of domestic animals on the road. This is great a challenge to consider those parameters and model the computer traffic simulation and also in the real traffic management.

\section{REFERENCES}

[1] Kotusevski G. and Hawick K. A. 2009. A Review of Traffic Simulation Software, Res. Lett. Inf. Math. Sci., Vol. 13, 35-54.

[2] Jain V., Sharma A., and Subramanian L. 2012. Road Traffic Congestion in the Developing world, Proc. of the 2nd ACM Symposium on Computing for Development, Article No. 11, March 11-12.

[3] TCL and IIM. 2012. Operational efficiency of freight transportation by road in India. Joint study by Transport 
Corporation of India Ltd and Indian Institute of Management Calcutta.

[4] MoRTH. 2014. Road Accidents in India 2013. Ministry of Road Transport and Highways, Government of India, $1-86$.

[5] Leksono C. Y. and Andriyana T. 2012. Roundabout microsimulation using SUMO. Linköping University, Sweden.

[6] “Annual Report 2014-2015”, Ministry of Road Transport and Highways, Government of India, pp. 1-154, 2015.

[7] Census. 2011. Cities having population 1 lakh and above, Census 2011. Office of the Registrar General \& Census Commissioner,

India. [http://www.censusindia.gov.in/2011-provresults/paper2/data_files/India2/Table_3_PR_UA_Citiee s_1Lakh_and_Above.pdf] (Last access on 15 April, 2015).

[8] Krajzewicz D, Erdmann J., Behrisch M., and Bieker L. 2012. Recent Development and Applications of SUMO Simulation of Urban MObility; International Journal on
Advances in Systems and Measurements, 5 (3\&4).128138.

[9] Krauss, S. 1998. Microscopic Modeling of Traffic Flow: Investigation of Collision Free Vehicle Dynamics. Koln: Universitat zu Koln.

[10] NETCONVERT.

http://sumo.dlr.de/wiki/NETCONVERT [Last access on $16 / 04 / 2015]$

[11] Open Street Map of Raipur. https://www.openstreetmap.org/\#map=16/21.2381/81.65 85 [Last access on 16/04/2015]

[12] OSM File Format. http://wiki.openstreetmap.org/wiki/OSM_file_formats [Last access on 16/04/2015]

[13] Google Map. https://www.google.co.in/maps/ [Last access on $16 / 04 / 2015$

[14] Maciejewski, M., 2010. A Comparison of Microscopic Traffic Flow Simulation System for an Urban Area. Transport Problem, 5(4). 